\title{
EIGENVALUE CONDITION NUMBERS AND A FORMULA OF BURKE, LEWIS AND OVERTON*
}

\author{
MICHAEL KAROW ${ }^{\dagger}$
}

\begin{abstract}
In a paper by Burke, Lewis and Overton, a first order expansion has been given for the minimum singular value of $A-z I, z \in \mathbb{C}$, about a nonderogatory eigenvalue $\lambda$ of $A \in \mathbb{C}^{n \times n}$. This note investigates the relationship of the expansion with the Jordan canonical form of $A$. Furthermore, formulas for the condition number of eigenvalues are derived from the expansion.
\end{abstract}

Key words. Eigenvalue condition numbers, Jordan canonical form, Singular values.

AMS subject classifications. 15A18, 65F35.

1. Introduction. By $\pi_{\Sigma}(A)$ we denote the product of the nonzero singular values of the matrix $A \in \mathbb{C}^{n \times m}$, counting multiplicities. For the zero matrix $0 \in \mathbb{C}^{n \times m}$ we set $\pi_{\Sigma}(0)=1$. If $A$ is square then $\Lambda(A)$ denotes the spectrum and $\pi_{\Lambda}(A)$ stands for the product of the nonzero eigenvalues, counting multiplicities. If all eigenvalues of $A$ are zero then we set $\pi_{\Lambda}(A)=1$. The subject of this note is the ratio

$$
q(A, \lambda):=\frac{\pi_{\Sigma}\left(A-\lambda I_{n}\right)}{\left|\pi_{\Lambda}\left(A-\lambda I_{n}\right)\right|}, \quad \lambda \in \Lambda(A) .
$$

In [1] the following first order expansion has been given for the function

$$
z \mapsto \sigma_{\min }\left(A-z I_{n}\right), z \in \mathbb{C},
$$

where $\sigma_{\min }(\cdot)$ denotes the minimum singular value and $I_{n}$ is the $n \times n$ identity matrix.

TheOREM 1.1. Let $\lambda \in \mathbb{C}$ be a nonderogatory eigenvalue of algebraic multiplicity $m$ of the matrix $A \in \mathbb{C}^{n \times n}$. Then

$$
\sigma_{\min }\left(A-z I_{n}\right)=\frac{|z-\lambda|^{m}}{q(A, \lambda)}+\mathcal{O}\left(|z-\lambda|^{m+1}\right), \quad z \in \mathbb{C} .
$$

The relevance of this result for the perturbation theory of eigenvalues is as follows. The closed $\epsilon$ - pseudospectrum of $A \in \mathbb{C}^{n \times n}$ with respect to the spectral norm, $\|\cdot\|$, is defined by

$$
\Lambda_{\epsilon}(A)=\left\{z \in \mathbb{C} \mid z \in \Lambda(A+\Delta), \Delta \in \mathbb{C}^{n \times n},\|\Delta\| \leq \epsilon\right\} .
$$

In words, $\Lambda_{\epsilon}(A)$ is the set of all eigenvalues of all matrices of the form $A+\Delta$ where the spectral norm of the perturbation $\Delta$ is bounded by $\epsilon>0$. It is well known [10] that

$$
\Lambda_{\epsilon}(A)=\left\{z \in \mathbb{C} \mid \sigma_{\min }(A-z I) \leq \epsilon\right\} .
$$

\footnotetext{
*Received by the editors 11 January 2006. Accepted for publication 25 April 2006. Handling Editor: Michael Neumann.

$\dagger$ Berlin University of Technology, Institute for Mathematics, Straße des 17.Juni 136, D-10623 Berlin, Germany (karow@math.TU-Berlin.de).
} 
Theorem 1.1 yields an estimate for the size of pseudospectra for small $\epsilon$ : Roughly speaking if $\epsilon$ is small enough then the connected component of $\Lambda_{\epsilon}(A)$ that contains the eigenvalue $\lambda$ is approximately a disk of radius $(q(A, \lambda) \epsilon)^{1 / m}$ about $\lambda$. It follows that $q(A, \lambda)^{1 / m}$ is the Hölder condition number of $\lambda$. We discuss this in detail in Section 4 .

However, the main concern of this note is to establish the relationship of $q(A, \lambda)$ with the Jordan decomposition of $A$. For a simple eigenvalue the relationship is as follows. Let $x, y \in \mathbb{C}^{n} \backslash\{0\}$ be a right and a left eigenvector of $A$ to the eigenvalue $\lambda$ respectively, i.e. $A x=\lambda x, y^{*} A=\lambda y^{*}$, where $y^{*}$ denotes the conjugate transpose of $y$. Then

$$
P=\left(y^{*} x\right)^{-1} x y^{*} \in \mathbb{C}^{n \times n}
$$

is a projection onto the one dimensional eigenspace $\mathbb{C} x$. The kernel of $P$ is the direct sum of all generalized eigenspaces belonging to the eigenvalues different from $\lambda$. As is well known [5, p.490],[3, p.202],[9, p.186], the condition number of $\lambda$ equals the norm of $P$. Combined with the considerations above this yields that

$$
q(A, \lambda)=\|P\| .
$$

In Section 3 we give an elementary proof of the identity (1.1) without using Theorem 1.1. Furthermore, we show that for a nondegoratory eigenvalue of algebraic multiplicity $m \geq 2$,

$$
q(A, \lambda)=\left\|N^{m-1}\right\|,
$$

where $N$ is the nilpotent operator associated with $\lambda$ in the Jordan decomposition of $A$. The formulas (1.1) and (1.2) are the main results of this note. The proofs also show that the assumption that $\lambda$ is nonderogatory is necessary.

The next section contains some preliminaries about the computation of the two products $\pi_{\Sigma}(A)$ and $\pi_{\Lambda}(A)$ and about the relationship of the Schur form of $A$ with the Jordan decomposition.

Throughout this note, $\|\cdot\|$ stands for the spectral norm.

2. Preliminaries. Below we list some easily verified properties of $\pi_{\Lambda}(A)$, the product of the nonzero eigenvalues of $A$, and of $\pi_{\Sigma}(A)$, the product of the nonzero singular values of $A$. In the sequel $A^{T}$ and $A^{*}$ denote the transpose and the conjugate transpose of $A$ respectively.

(a) If $A \in \mathbb{C}^{n \times n}$ is nonsingular then $\pi_{\Lambda}(A)=\operatorname{det}(A)$.

(b) For any $A \in \mathbb{C}^{n \times n}: \pi_{\Lambda}\left(A^{T}\right)=\pi_{\Lambda}(A)$ and $\pi_{\Lambda}\left(A^{*}\right)=\overline{\pi_{\Lambda}(A)}$.

(c) Let $S \in \mathbb{C}^{n \times n}$ be nonsingular. Then for any $A \in \mathbb{C}^{n \times n}, \pi_{\Lambda}\left(S A S^{-1}\right)=\pi_{\Lambda}(A)$.

(d) Let $A_{11} \in \mathbb{C}^{n \times n}, A_{22} \in \mathbb{C}^{m \times m}$ and $A_{12} \in \mathbb{C}^{n \times m}$. Then

$$
\pi_{\Lambda}\left(\left[\begin{array}{cc}
A_{11} & A_{12} \\
0 & A_{22}
\end{array}\right]\right)=\pi_{\Lambda}\left(A_{11}\right) \pi_{\Lambda}\left(A_{22}\right) .
$$

(e) For any $A \in \mathbb{C}^{n \times m}, \pi_{\Sigma}(A)^{2}=\pi_{\Lambda}\left(A^{*} A\right)=\pi_{\Lambda}\left(A A^{*}\right)$. 
(f) If $A \in \mathbb{C}^{n \times n}$ is nonsingular then $\pi_{\Sigma}(A)=|\operatorname{det}(A)|=\left|\pi_{\Lambda}(A)\right|$.

(g) Let $U \in \mathbb{C}^{n \times n}$ and $V \in \mathbb{C}^{m \times m}$ be unitary. Then for any $A \in \mathbb{C}^{n \times m}$, $\pi_{\Sigma}(U A V)=\pi_{\Sigma}(A)$.

In the next section we need the lemmas below.

Lemma 2.1. Let $M \in \mathbb{C}^{n \times n}$ be nonsingular, $X \in \mathbb{C}^{m \times n}$ and $Y=X M^{-1}$. Then

$$
\pi_{\Sigma}\left(\left[\begin{array}{c}
M \\
X
\end{array}\right]\right)=\pi_{\Sigma}(M) \sqrt{\operatorname{det}\left(I_{n}+Y^{*} Y\right)} .
$$

Proof. We have

$$
\begin{aligned}
\pi_{\Sigma}\left(\left[\begin{array}{c}
M \\
X
\end{array}\right]\right)^{2} & =\pi_{\Lambda}\left(\left[\begin{array}{ll}
M^{*} & X^{*}
\end{array}\right]\left[\begin{array}{c}
M \\
X
\end{array}\right]\right) \\
& =\operatorname{det}\left(M^{*} M+X^{*} X\right) \\
& =\operatorname{det}\left(M^{*}\left(I_{n}+Y^{*} Y\right) M\right) \\
& =\operatorname{det}\left(M^{*}\right) \operatorname{det}(M) \operatorname{det}\left(I_{n}+Y^{*} Y\right) \\
& =\pi_{\Sigma}(M)^{2} \operatorname{det}\left(I_{n}+Y^{*} Y\right) .
\end{aligned}
$$

Lemma 2.2. Let $Y \in \mathbb{C}^{m \times n}$. Then $\left\|I_{n}+Y^{*} Y\right\|=\left\|I_{m}+Y Y^{*}\right\|$ and $\operatorname{det}\left(I_{n}+\right.$ $\left.Y^{*} Y\right)=\operatorname{det}\left(I_{m}+Y Y^{*}\right)$.

Proof. The case $Y=0$ is trivial. Let $Y \neq 0$. The matrices $Y$ and $Y^{*}$ have the same nonzero singular values $\sigma_{1} \geq \sigma_{2} \geq \ldots \geq \sigma_{p}>0$ say. The eigenvalues different from 1 of both $I_{n}+Y^{*} Y$ and $I_{m}+Y Y^{*}$ are $1+\sigma_{1}^{2} \geq 1+\sigma_{2}^{2} \ldots \geq 1+\sigma_{p}^{2}$. Thus $\left\|I_{n}+Y^{*} Y\right\|=\left\|I_{m}+Y Y^{*}\right\|=1+\sigma_{1}^{2}$ and $\operatorname{det}\left(I_{n}+Y^{*} Y\right)=\operatorname{det}\left(I_{m}+Y Y^{*}\right)=$ $\prod_{k=1}^{p}\left(1+\sigma_{k}^{2}\right)$.

We proceed with remarks on the Jordan decomposition. Let $\lambda_{1}, \ldots, \lambda_{\kappa}$ be the pairwise different eigenvalues of $A \in \mathbb{C}^{n \times n}$. Let $\mathcal{X}_{j}=\operatorname{ker}\left(A-\lambda_{j} I_{n}\right)^{n}$ be the generalized eigenspaces. By the Jordan decomposition theorem we have

$$
A=\sum_{j=1}^{\kappa}\left(\lambda_{j} P_{j}+N_{j}\right)
$$

where $P_{1}, \ldots, P_{\kappa} \in \mathbb{C}^{n \times n}$ are the projectors of direct decomposition $\mathbb{C}^{n}=\bigoplus_{j=1}^{\kappa} \mathcal{X}_{j}$, i.e.

$$
P_{j}^{2}=P_{j}, \quad \operatorname{range}\left(P_{j}\right)=\mathcal{X}_{j}, \quad \operatorname{ker}\left(P_{j}\right)=\bigoplus_{k=1, k \neq j}^{\kappa} \mathcal{X}_{k},
$$

and $N_{1}, \ldots, N_{\kappa} \in \mathbb{C}^{n \times n}$ are the nilpotent matrices $N_{j}=\left(A-\lambda_{j} I_{n}\right) P_{j}$. The eigenvalue $\lambda_{j}$ is said to be

- semisimple (nondefective) if $\mathcal{X}_{j}=\operatorname{ker}\left(A-\lambda_{j} I_{n}\right)$,

- simple if $\operatorname{dim} \mathcal{X}_{j}=1$,

- nonderogatory if $\operatorname{dim} \operatorname{ker}\left(A-\lambda_{j} I_{n}\right)=1$. 
In the following $m$ denotes the algebraic multiplicity of $\lambda_{j}$. Note that if $m \geq 2$ then $\lambda_{j}$ is nonderogatory if and only if $N_{j}^{m-1} \neq 0$. We now recall how to obtain the operators $P_{j}$ and $N_{j}$ from a Schur form of $A$. We only consider the nontrivial case that $A$ has at least two different eigenvalues. By the Schur decomposition theorem there exists a unitary matrix $U \in \mathbb{C}^{n \times n}$ such that

$$
U^{*} A U=\left[\begin{array}{cc}
\lambda_{j} I_{m}+T & A_{12} \\
0 & A_{22}
\end{array}\right]
$$

where $A_{12} \in \mathbb{C}^{m \times(n-m)}, A_{22} \in \mathbb{C}^{(n-m) \times(n-m)}, \Lambda\left(A_{22}\right)=\Lambda(A) \backslash\left\{\lambda_{j}\right\}$ and $T \in \mathbb{C}^{n \times n}$ is strictly upper triangular,

$$
T=\left[\begin{array}{ccccc}
0 & t_{12} & \ldots & \cdots & t_{1 m} \\
& \ddots & t_{23} & & \vdots \\
& & \ddots & \ddots & \vdots \\
& & & \ddots & t_{m-1, m} \\
& & & & 0
\end{array}\right] .
$$

If $m=1$ (i.e. $\lambda_{j}$ is simple) then $T$ is the $1 \times 1$ zero matrix. Since the spectra of $T$ and $A_{22}-\lambda_{j} I_{n-m}$ are disjoint the Sylvester equation

$$
R\left(A_{22}-\lambda_{j} I_{n-m}\right)-T R=A_{12} .
$$

has a unique solution $R \in \mathbb{C}^{m \times(n-m)}$.

Proposition 2.3. With the notation above the projector onto the generalized eigenspace and the nilpotent operator associated with $\lambda_{j}$ are given by

$$
P_{j}=U\left[\begin{array}{cc}
I_{m} & -R \\
0 & 0
\end{array}\right] U^{*}, \quad \text { and } \quad N_{j}=U\left[\begin{array}{cc}
T & -T R \\
0 & 0
\end{array}\right] U^{*} .
$$

For any integer $\ell \geq 1$ we have

$$
N_{j}^{\ell}=U\left[\begin{array}{cc}
T^{\ell} & -T^{\ell} R \\
0 & 0
\end{array}\right] U^{*}
$$

The spectral norms of $P_{j}$ and of $N_{j}^{\ell}$ satisfy

$$
\begin{aligned}
\left\|P_{j}\right\| & =\left\|I_{m}+R R^{*}\right\|^{1 / 2} \\
\left\|N_{j}^{\ell}\right\| & =\left\|T^{\ell}\left(I_{m}+R R^{*}\right)\left(T^{*}\right)^{\ell}\right\|^{1 / 2} .
\end{aligned}
$$

Proof. Let $X_{1}:=U\left[\begin{array}{c}I_{m} \\ 0\end{array}\right] \in \mathbb{C}^{n \times m}, X_{2}:=U\left[\begin{array}{c}R \\ I_{n-m}\end{array}\right] \in \mathbb{C}^{n \times(n-m)}$. Then obviously $\mathbb{C}^{n}=\operatorname{range}\left(X_{1}\right) \oplus \operatorname{range}\left(X_{2}\right)$ and

$$
A X_{1}=X_{1}\left(\lambda_{j} I_{m}+T\right) .
$$


Furthermore, (2.2) yields that

$$
A X_{2}=X_{2} A_{22} .
$$

Hence, range $\left(X_{1}\right)$ and range $\left(X_{2}\right)$ are complementary invariant subspaces of $A$. The relations (2.6) and (2.7) imply that for any $\lambda \in \mathbb{C}$ and any integer $\ell \geq 1$,

$$
\begin{aligned}
& \left(A-\lambda I_{n}\right)^{\ell} X_{1}=X_{1}\left(\left(\lambda_{j}-\lambda\right) I_{m}+T\right)^{\ell}, \\
& \left(A-\lambda I_{n}\right)^{\ell} X_{2}=X_{2}\left(A_{22}-\lambda I_{n-m}\right)^{\ell} .
\end{aligned}
$$

Using this and the fact that $\lambda_{j} \notin \Lambda\left(A_{22}\right)$ it is easily verified that range $\left(X_{1}\right)=\operatorname{ker}(A-$ $\left.\lambda_{j} I_{n}\right)^{n}$ and range $\left(X_{2}\right)=\bigoplus_{k=1, k \neq j}^{\kappa} \operatorname{ker}\left(A-\lambda_{k} I_{n}\right)^{n}$. The matrix

$$
P_{j}=U\left[\begin{array}{cc}
I_{m} & -R \\
0 & 0
\end{array}\right] U^{*}
$$

satisfies $P_{j}^{2}=P_{j}, P_{j} X_{1}=X_{1}$ and $P_{j} X_{2}=0$. Hence, $P_{j}$ is the Jordan projector onto the generalized eigenspace $\operatorname{ker}\left(A-\lambda_{j} I_{n}\right)^{n}$. For the associated nilpotent matrix $N_{j}$ one obtains

$$
N_{j}=\left(A-\lambda_{j} I_{n}\right) P_{j}=U\left[\begin{array}{cc}
T & -T R \\
0 & 0
\end{array}\right] U^{*}
$$

The formulas (2.3), (2.4) and (2.5) are immediate from (2.9) and (2.10).

We give an expression for $\left\|N_{j}^{m-1}\right\|$ which is a bit more explicit than formula (2.5).

First note that if $\lambda_{j}$ has algebraic multiplicity $m \geq 2$ then

$$
T^{m-1}=\left[\begin{array}{cccc}
0 & \ldots & 0 & \tau \\
\vdots & & \vdots & 0 \\
\vdots & & \vdots & \vdots \\
0 & \ldots & 0 & 0
\end{array}\right], \quad \text { where } \tau=\prod_{k=1}^{m-1} t_{k, k+1} .
$$

Let $e_{m}^{T}=\left[\begin{array}{lll}0 \ldots 0 & 1\end{array}\right]^{T} \in \mathbb{C}^{m}$ and $r=e_{m}^{T} R$. Then $r$ is the lower row of $R$. Since the lower row of $T R$ is zero it follows from the Sylvester equation (2.2) that

$$
r=e_{m}^{T} A_{12}\left(A_{22}-\lambda_{j} I_{m}\right)^{-1} .
$$

From (2.3) or (2.5) we obtain

Proposition 2.4. Suppose $\lambda_{j}$ has algebraic multiplicity $m \in\{2, \ldots, n-1\}$. Then

$$
\left\|N_{j}^{m-1}\right\|=|\tau| \sqrt{1+\|r\|^{2}}
$$


3. Main result. We are now in a position to state and prove our main result on the ratio

$$
q\left(A, \lambda_{j}\right)=\frac{\pi_{\Sigma}\left(A-\lambda_{j} I_{n}\right)}{\left|\pi_{\Lambda}\left(A-\lambda_{j} I_{n}\right)\right|}, \quad \lambda_{j} \in \Lambda(A) .
$$

Theorem 3.1. Let $\lambda_{j} \in \mathbb{C}$ be an eigenvalue of $A \in \mathbb{C}^{n \times n}$. Let $P_{j}$ and $N_{j}$ be the eigenprojector and the nilpotent operator associated with $\lambda_{j}$. Then the following holds.

(a) If $\lambda_{j}$ is a semisimple eigenvalue then $q\left(A, \lambda_{j}\right)=\pi_{\Sigma}\left(P_{j}\right)$.

(b) If $\lambda_{j}$ is a simple eigenvalue then $q\left(A, \lambda_{j}\right)=\left\|P_{j}\right\|$.

(c) If $\lambda_{j}$ is a nonderogatory eigenvalue of algebraic multiplicity $m \geq 2$ then

$$
q\left(A, \lambda_{j}\right)=\left\|N_{j}^{m-1}\right\| .
$$

Proof. First, we treat the case that $A$ has at least two different eigenvalues. In view of Proposition 2.3 and since the products $\pi_{\Sigma}\left(A-\lambda_{j} I_{n}\right), \pi_{\Lambda}\left(A-\lambda_{j} I_{n}\right)$ are invariant under unitary similarity transformations we may assume that

$$
A=\left[\begin{array}{cc}
\lambda_{j} I_{m}+T & A_{12} \\
0 & A_{22}
\end{array}\right], \quad P_{j}=\left[\begin{array}{cc}
I_{m} & -R \\
0 & 0
\end{array}\right],
$$

where $\Lambda\left(A_{22}\right)=\Lambda(A) \backslash\left\{\lambda_{j}\right\}, T \in \mathbb{C}^{n \times n}$ is strictly upper triangular and $R \in \mathbb{C}^{m \times(n-m)}$ is the solution of the Sylvester equation $R\left(A_{22}-\lambda_{j} I_{n-m}\right)-T R=A_{12}$.

(a). Suppose $\lambda_{j}$ is semisimple. Then $T=0$ and $R\left(A_{22}-\lambda_{j} I_{n-m}\right)=A_{12}$. Thus,

$$
\begin{aligned}
\left(A-\lambda_{j} I_{n}\right)^{*}\left(A-\lambda_{j} I_{n}\right) & =\left[\begin{array}{cc}
0 & 0 \\
0 & \left(A_{22}-\lambda_{j} I_{n-m}\right)^{*}\left(A_{22}-\lambda_{j} I_{n-m}\right)+A_{12}^{*} A_{12}
\end{array}\right] \\
& =\left[\begin{array}{cc}
0 & 0 \\
0 & \left(A_{22}-\lambda_{j} I_{n-m}\right)^{*}\left(I_{n-m}+R^{*} R\right)\left(A_{22}-\lambda_{j} I_{n-m}\right)
\end{array}\right] .
\end{aligned}
$$

Thus

$$
\begin{aligned}
\pi_{\Sigma}\left(A-\lambda_{j} I_{n}\right)^{2} & =\pi_{\Lambda}\left(\left(A-\lambda_{j} I_{n}\right)^{*}\left(A-\lambda_{j} I_{n}\right)\right) \\
& =\operatorname{det}\left(\left(A_{22}-\lambda_{j} I_{n-m}\right)^{*}\left(I_{n-m}+R^{*} R\right)\left(A_{22}-\lambda_{j} I_{n-m}\right)\right) \\
& =\left|\operatorname{det}\left(A_{22}-\lambda_{j} I_{n-m}\right)\right|^{2} \operatorname{det}\left(I_{n-m}+R^{*} R\right) \\
& =\left|\pi_{\Lambda}\left(A-\lambda_{j} I_{n}\right)\right|^{2} \operatorname{det}\left(I_{n-m}+R^{*} R\right) .
\end{aligned}
$$

Furthermore we have $P_{j} P_{j}^{*}=\left[\begin{array}{cc}I_{m}+R R^{*} & 0 \\ 0 & 0\end{array}\right]$ and hence

$$
\pi_{\Sigma}\left(P_{j}\right)^{2}=\operatorname{det}\left(I_{m}+R R^{*}\right)=\operatorname{det}\left(I_{n-m}+R^{*} R\right) .
$$

The latter equation holds by Lemma 2.2. By combining (3.3) and (3.4) we obtain $(a)$. (b). If $m=1$ then $P_{j}$ has rank 1 and hence, $\pi_{\Sigma}\left(P_{j}\right)=\left\|P_{j}\right\|$. Thus (b) follows from (a). 
(c) Suppose $m \geq 2$ and $\lambda_{j}$ is nonderogatory. Then $T=\left[\begin{array}{c}0 \\ \vdots \\ 0 \\ \ldots\end{array}\right]$, where $D \in$ $\mathbb{C}^{(m-1) \times(m-1)}$ is upper triangular and nonsingular. In the following we write $A_{12}=$ $\left[\begin{array}{c}\tilde{A} \\ a\end{array}\right]$, where $a$ is the lower row of $A_{12}$. Let $r$ denote the lower row of $R$. By Formula (2.11) we have

$$
r=a\left(A_{22}-\lambda_{j} I\right)^{-1}
$$

Let us determine $\pi_{\Sigma}(A)$. Since removing of a column of zeros and a permutation of rows does not change the nonzero singular values of a matrix we have

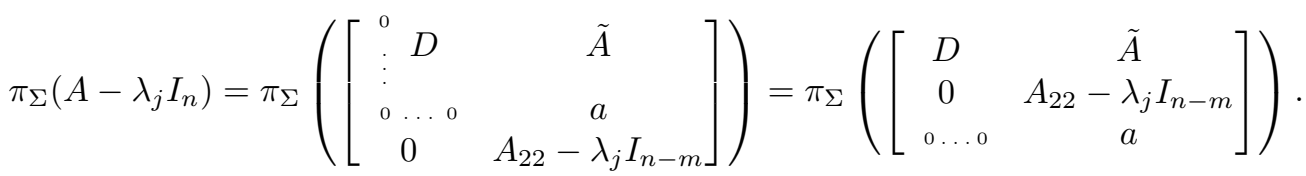

Lemma 2.1 yields

$$
\begin{aligned}
\pi_{\Sigma}\left(\left[\begin{array}{cc}
D & \tilde{A} \\
0 & A_{22}-\lambda_{j} I_{n-m} \\
0 \ldots 0 & a
\end{array}\right]\right) & =\pi_{\Sigma}\left(\left[\begin{array}{cc}
D & \tilde{A} \\
0 & A_{22}-\lambda_{j} I
\end{array}\right]\right) \sqrt{\operatorname{det}\left(1+y y^{*}\right)} \\
& =\left|\operatorname{det}(D) \operatorname{det}\left(A_{22}-\lambda_{j} I\right)\right| \sqrt{1+\|y\|^{2}} \\
& =\left|\pi_{\Lambda}\left(A-\lambda_{j} I\right)\right||\operatorname{det}(D)| \sqrt{1+\|y\|^{2}}
\end{aligned}
$$

where

$$
y=\left[\begin{array}{ll}
0 \ldots 0 & a
\end{array}\right]\left[\begin{array}{cc}
D & \tilde{A} \\
0 & A_{22}-\lambda_{j} I
\end{array}\right]^{-1}
$$

From (3.5) it follows that $y=\left[\begin{array}{ll}0 \ldots 0 & r\end{array}\right]$ and hence, $\|y\|=\|r\|$. In summary,

$$
\pi_{\Sigma}\left(A-\lambda_{j} I_{n}\right)=\left|\pi_{\Lambda}\left(A-\lambda_{j} I_{n}\right)\right||\operatorname{det}(D)| \sqrt{1+\|r\|^{2}} .
$$

But $|\operatorname{det}(D)| \sqrt{1+\|r\|^{2}}=\left\|N_{j}^{m-1}\right\|$ by Proposition 2.4. Hence, (c) holds.

Finally, we treat the case that $\lambda_{1}$ is the only eigenvalue of $A$. Let $U^{*} A U=\lambda_{1} I_{n}+T$ be a Schur decomposition. The eigenprojection is $P_{1}=I_{n}$ and the nilpotent operator is $N_{1}=A-\lambda_{1} I_{n}=U T U^{*}$. Since all eigenvalues of $A-\lambda_{1} I_{n}$ are zero we have $\pi_{\Lambda}\left(A-\lambda_{1} I_{n}\right)=1$ by definition. If $\lambda_{1}$ is semisimple then also $\pi_{\Sigma}\left(A-\lambda_{1} I_{n}\right)=\pi_{\Sigma}(0)=$ 1. Hence, $q\left(A, \lambda_{1}\right)=1=\pi_{\Sigma}\left(P_{1}\right)$. Suppose $n \geq 2$ and $\lambda_{1}$ is nonderogatory. Then

$$
q\left(A, \lambda_{1}\right)=\pi_{\Sigma}\left(A-\lambda_{1} I_{n}\right)=\pi_{\Sigma}(T)=|\operatorname{det}(D)|=\left\|T^{n-1}\right\|=\left\|N_{1}^{n-1}\right\|,
$$

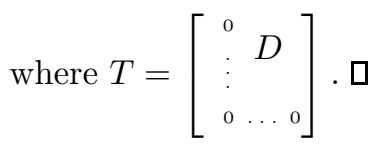


4. Condition numbers. In this section we show that $q(A, \lambda)^{1 / m}$ equals the Hölder condition number of the nonderogatory eigenvalue $\lambda$ of algebraic multiplicity $m$. To this end we introduce some additional notation. By $\mathcal{D}_{\lambda}(r)$ we denote the closed disk of radius $r>0$ about $\lambda \in \mathbb{C}$. If $\lambda \in \Lambda(A), A \in \mathbb{C}^{n \times n}$, then $\mathcal{C}_{\lambda}(\epsilon)$ denotes the connected component of the $\epsilon$-pseudospectrum, $\Lambda_{\epsilon}(A)$, that contains $\lambda$. We define

$$
\begin{aligned}
& R_{\lambda}^{+}(\epsilon):=\inf \left\{r>0 \mid \mathcal{C}_{\lambda}(\epsilon) \subseteq \mathcal{D}_{\lambda}(r)\right\} \\
& R_{\lambda}^{-}(\epsilon):=\sup \left\{r>0 \mid \mathcal{D}_{\lambda}(r) \subseteq \mathcal{C}_{\lambda}(\epsilon)\right\}
\end{aligned}
$$

Then

$$
\mathcal{D}_{\lambda}\left(R_{\lambda}^{-}(\epsilon)\right) \subseteq \mathcal{C}_{\lambda}(\epsilon) \subseteq \mathcal{D}_{\lambda}\left(R_{\lambda}^{+}(\epsilon)\right) .
$$

TheOREm 4.1. Let $\lambda \in \Lambda(A)$ be a nonderogatory eigenvalue of algebraic multiplicity $m$. Then

$$
R_{\lambda}^{ \pm}(\epsilon)=q(A, \lambda)^{1 / m} \epsilon^{1 / m}+o\left(\epsilon^{1 / m}\right) .
$$

The proof uses Theorem 1.1 and the lemma below.

Lemma 4.2. Let $U \subseteq \mathbb{C}^{n}$ be an open neighborhood of $z_{0} \in \mathbb{C}^{n}$. Let $f, g$ : $U \rightarrow[0, \infty)$ be continuous functions. For $\epsilon \geq 0$ let $S_{f}(\epsilon)$ and $S_{g}(\epsilon)$ denote the connected component containing $z_{0}$ of the sublevel set $\{z \in U \mid f(z) \leq \epsilon\}$ and $\{z \in U \mid g(z) \leq \epsilon\}$ respectively. Assume that $0=g\left(z_{0}\right)$ is an isolated zero of $g$, and

$$
\lim _{z \rightarrow z_{0}} \frac{f(z)}{g(z)}=1
$$

Then there exists an $\epsilon_{0}>0$ and functions $h_{ \pm}:\left[0, \epsilon_{0}\right] \rightarrow[0, \infty)$ with $\lim _{\epsilon \rightarrow 0} h_{ \pm}(\epsilon)=1$ such that for all $\epsilon \in\left[0, \epsilon_{0}\right]$,

$$
S_{g}\left(h_{-}(\epsilon) \epsilon\right) \subseteq S_{f}(\epsilon) \subseteq S_{g}\left(h_{+}(\epsilon) \epsilon\right) .
$$

We postpone the proof of the lemma to the end of this section.

Proof of Theorem 4.1: Let in Lemma 4.2, $z_{0}=\lambda$ and

$$
f(z)=\sigma_{\min }\left(A-z I_{n}\right), \quad g(z)=\frac{|z-\lambda|^{m}}{q(A, \lambda)}, \quad z \in \mathbb{C} .
$$

Then $S_{f}(\epsilon)=\mathcal{C}_{\lambda}(\epsilon)$ and $S_{g}(\epsilon)=\mathcal{D}_{\lambda}\left((q(A, \lambda) \epsilon)^{1 / m}\right)$. Theorem 1.1 yields $\lim _{z \rightarrow \lambda} \frac{f(z)}{g(z)}=$ 1. Hence, by the lemma there are functions $h_{ \pm}$with $\lim _{\epsilon \rightarrow 0} h_{ \pm}(\epsilon)=1$ and

$$
\mathcal{D}_{\lambda}\left(\left(q(A, \lambda) h_{-}(\epsilon) \epsilon\right)^{1 / m}\right) \subseteq \mathcal{C}_{\lambda}(\epsilon) \subseteq \mathcal{D}_{\lambda}\left(\left(q(A, \lambda) h_{+}(\epsilon) \epsilon\right)^{1 / m}\right) .
$$

This shows (4.1). $\mathrm{\square}$

Now, we give the definition for the Hölder condition number of an eigenvalue of arbitrary multiplicity (see [2]). For $\lambda \in \mathbb{C}, m \in \mathbb{N}$ and $\widetilde{A} \in \mathbb{C}^{n \times n}$ we set

$$
d_{m}(\widetilde{A}, \lambda):=\min \left\{r \geq 0 \mid \mathcal{D}_{\lambda}(r) \text { contains at least } m \text { eigenvalues of } \widetilde{A}\right\} .
$$


If $\lambda$ is an eigenvalue of $A \in \mathbb{C}^{n \times n}$ of algebraic multiplicity $m$ then the Hölder condition number of $\lambda$ to the order $\alpha>0$ is defined by

$$
\operatorname{cond}_{\alpha}(A, \lambda)=\lim _{\epsilon \searrow 0} \sup _{\|\Delta\| \leq \epsilon} \frac{d_{m}(A+\Delta, \lambda)}{\|\Delta\|^{\alpha}} .
$$

It is easily seen that $0 \neq \operatorname{cond}_{\alpha}(A, \lambda) \neq \infty$ for at most one order $\alpha>0$.

TheOREM 4.3. Let $\lambda \in \Lambda(A)$ be a nonderogatory eigenvalue of multiplicity $m$. Then

$$
\operatorname{cond}_{1 / m}(A, \lambda)=q(A, \lambda)^{1 / m}= \begin{cases}\|P\| & \text { if } m=1 \\ \left\|N^{m-1}\right\|^{1 / m} & \text { otherwise }\end{cases}
$$

where $P \in \mathbb{C}^{n \times n}$ is the eigenprojector onto the generalized eigenspace $\operatorname{ker}\left(A-\lambda I_{n}\right)^{m}$, and $N=\left(A-\lambda I_{m}\right) P$.

Proof. Let $\Delta \in \mathbb{C}^{n \times n}$ with $\|\Delta\| \leq \epsilon$. Then the continuity of eigenvalues yields, that for any $t \in[0,1]$ at least $m$ eigenvalues of $A+t \Delta$ are contained in $\mathcal{C}_{\lambda}(\epsilon)$ counting multiplicities. Hence

$$
d_{m}(A+\Delta, \lambda) \leq R_{\lambda}^{+}(\epsilon)=q(A, \lambda)^{1 / m} \epsilon^{1 / m}+o\left(\epsilon^{1 / m}\right) .
$$

By letting $\epsilon=\|\Delta\|$ we obtain that for all $\Delta \in \mathbb{C}^{n \times n}$,

$$
\frac{d_{m}(A+\Delta, \lambda)}{\|\Delta\|^{1 / m}} \leq q(A, \lambda)^{1 / m}+o\left(\|\Delta\|^{1 / m}\right)\|\Delta\|^{-(1 / m)} .
$$

This yields

$$
\operatorname{cond}_{1 / m}(A, \lambda) \leq q(A, \lambda)^{1 / m} .
$$

Let $r>0$ be such that $\mathcal{D}_{\lambda}(r) \cap \Lambda(A)=\{\lambda\}$. Then by the continuity of eigenvalues there is an $\epsilon_{0}$ such that the following holds for all $\epsilon<\epsilon_{0}$,

(a) $\mathcal{D}_{\lambda}(r) \cap \Lambda_{\epsilon}(A)=\mathcal{C}_{\lambda}(\epsilon)$.

(b) For any $\Delta \in \mathbb{C}^{n \times n}$ with $\|\Delta\| \leq \epsilon$, the set $\mathcal{C}_{\lambda}(\epsilon)$ contains precisely $m$ eigenvalues of $A+\Delta$ counting multiplicities.

Let $\epsilon<\epsilon_{0}$ and let $z_{\epsilon} \in \mathbb{C}$ be a boundary point of $\mathcal{C}_{\lambda}(\epsilon)$. Then $\sigma_{\min }\left(A-z_{\epsilon} I_{n}\right)=\epsilon$. Let $\Delta_{\epsilon}=-\epsilon u v^{*}$, where $u, v \in \mathbb{C}^{n}$ is a pair of normalized left and right singular vectors of $A-z_{\epsilon} I_{n}$ belonging to the minimum singular value, i.e.

$$
\left(A-z_{\epsilon} I_{n}\right) v=\epsilon u, \quad u^{*}\left(A-z_{\epsilon} I_{n}\right)=\epsilon v^{*}, \quad\|u\|=\|v\|=1 .
$$

Then $\left\|\Delta_{\epsilon}\right\|=\epsilon$ and $z_{\epsilon} \in \Lambda\left(A+\Delta_{\epsilon}\right)$ since $\left(A+\Delta_{\epsilon}\right) v=z_{\epsilon} v$. Thus, by $(a)$ and $(b)$,

$$
\begin{aligned}
d_{m}\left(A+\Delta_{\epsilon}, \lambda\right) & \geq\left|z_{\epsilon}-\lambda\right| \\
& \geq R_{\lambda}^{-}(\epsilon) \\
& =q(A, \lambda)^{1 / m} \epsilon^{1 / m}+o\left(\epsilon^{1 / m}\right) .
\end{aligned}
$$


and therefore

$$
\frac{d_{m}\left(A+\Delta_{\epsilon}, \lambda\right)}{\left\|\Delta_{\epsilon}\right\|^{1 / m}} \geq q(A, \lambda)^{1 / m}+o\left(\epsilon^{1 / m}\right) \epsilon^{-(1 / m)} .
$$

Hence, $\operatorname{cond}_{1 / m}(A, \lambda) \geq q(A, \lambda)^{1 / m}$. $\mathrm{P}$

REMARK 4.4. In [7] (see also [2,4]) the following generalization of Theorem 4.3 has been shown. Let $\lambda$ be an arbitrary eigenvalue of $A$. If $\lambda$ is semisimple then

$$
\operatorname{cond}_{1}(A, \lambda)=\|P\| \text {. }
$$

If $\lambda$ is not semisimple then

$$
\operatorname{cond}_{1 / m}(A, \lambda)=\left\|N^{m-1}\right\|^{1 / m},
$$

where $m$ denotes the index of nilpotency of $N$, i.e. $N^{m}=0, N^{m-1} \neq 0$.

Proof of Lemma 4.2: By $B_{r}$ we denote the closed ball of radius $r>0$ about $z_{0}$. The condition that $z_{0}$ is an isolated zero of $g$ combined with (4.2) yields that $z_{0}$ is also an isolated zero of $f$. Hence, there is an $r_{0}>0$ such that $f(z)>0$ for all $z \in B_{r_{0}} \backslash\left\{z_{0}\right\}$. This implies that $\epsilon_{r}:=\min _{z \in \partial B_{r}} f(z)>0$ for any $r \in\left(0, r_{0}\right]$. If $\epsilon<\epsilon_{r}$ then $\partial B_{r}$ does not intersect the sublevel sets $\{z \in U \mid f(z) \leq \epsilon\}$. Thus $S_{f}(\epsilon)$ is contained in the interior of $B_{r}$. Note that $S_{f}(\epsilon)$ being a connected component of a closed set is closed. It follows that $S_{f}(\epsilon)$ is compact if $\epsilon<\epsilon_{r_{0}}$. Now, let

$$
\phi_{ \pm}(z):= \begin{cases}\left(1 \pm\left\|z-z_{0}\right\|\right) \frac{g(z)}{f(z)} & z \in B_{r_{0}} \backslash\left\{z_{0}\right\}, \\ 1, & z=z_{0} .\end{cases}
$$

Condition (4.2) yields that the functions $\phi_{ \pm}: U \rightarrow \mathbb{R}$ are continuous. For $\epsilon<\epsilon_{r_{0}}$ let

$$
h_{-}(\epsilon):=\min _{z \in S_{f}(\epsilon)} \phi_{-}(z), \quad h_{+}(\epsilon):=\max _{z \in S_{f}(\epsilon)} \phi_{+}(z) .
$$

Then we have for all $\epsilon<\epsilon_{r}$,

$$
\min _{z \in B_{r}} \phi_{ \pm}(z) \leq h_{ \pm}(\epsilon) \leq \max _{z \in B_{r}} \phi_{ \pm}(z) .
$$

As $r$ tends to 0 the max and the min tend to $\phi_{ \pm}\left(z_{0}\right)=1$. This yields $\lim _{\epsilon \rightarrow 0} h_{ \pm}(\epsilon)=1$. If $z \in \partial S_{f}(\epsilon)$ then $f(z)=\epsilon$ and $g(z)>\left(1-\left\|z-z_{0}\right\|\right) \frac{g(z)}{f(z)} f(z) \geq h_{-}(\epsilon) \epsilon$. Thus $\partial S_{f}(\epsilon)$ does not intersect $E:=\left\{z \in U \mid g(z) \leq h_{-}(\epsilon) \epsilon\right\}$. Thus $S_{g}\left(h_{-}(\epsilon) \epsilon\right)$ being a connected component of $E$ is either contained in the interior of $S_{f}(\epsilon)$ or in the complement of $S_{f}(\epsilon)$. The latter is impossible since $z_{0} \in S_{f}(\epsilon) \cap S_{g}\left(h_{-}(\epsilon) \epsilon\right)$. Hence, $S_{g}\left(h_{-}(\epsilon) \epsilon\right) \subset S_{f}(\epsilon)$. This proves the first inclusion in (4.3). To prove the second suppose $z_{0} \neq z \in \partial S_{g}\left(h_{+}(\epsilon) \epsilon\right) \cap S_{f}(\epsilon)$. Then $g(z)=h_{+}(\epsilon) \epsilon$ and $0<f(z) \leq \epsilon$. Hence $g(z) / f(z) \geq h_{+}(\epsilon)$, a contradiction. Thus $S_{f}(\epsilon)$ is contained in the interior of $S_{g}\left(h_{+}(\epsilon) \epsilon\right)$. 


\section{REFERENCES}

[1] J. V. Burke, A. S. Lewis, and M. L. Overton. Optimization and pseudospectra, with applications to robust stability. SIAM J. Matrix Anal. Appl., 25:80-104, 2003.

[2] F. Chaitin-Chatelin, A. Harrabi, and A. Ilahi. About Hölder condition numbers and the stratification diagram for defective eigenvalues. Math. Comput. Simul., 54(4-5):397-402, 2000.

[3] G. H. Golub and C. F. Van Loan. Matrix Computations. Johns Hopkins University Press, Baltimore, 1988.

[4] A. Harrabi. Pseudospectres d'Operateurs Intégraux et Différentiels: Application a la Physique Mathematique. Thesis. Universite des Sciences Sociales de Toulouse, May 1998.

[5] D. Hinrichsen and A. J. Pritchard. Mathematical Systems Theory I. Modelling, State Space Analysis, Stability and Robustness. Springer-Verlag, Berlin, 2005.

[6] R. A. Horn, and C. R. Johnson. Matrix analysis. Cambridge University Press, Cambridge, 1985.

[7] M. Karow. Geometry of spectral value sets. Ph.D. thesis. University of Bremen, Germany, July 2003.

[8] J. Moro, J. V. Burke, and M. L. Overton. On the Lidskii-Vishik-Lyusternik perturbation theory for eigenvalues of matrices with arbitrary Jordan structure. SIAM J. Matrix Anal. Appl., 18(4):793-817, 1997.

[9] G. W. Stewart and J. Sun. Matrix Perturbation Theory. Academic Press, San Diego, 1990.

[10] L. N. Trefethen. Pseudospectra of linear operators. SIAM Review, 39:383-406, 1997.

[11] L. N. Trefethen and M. Embree. Spectra and Pseudospectra. The behavior of nonnormal matrices and operators. Princeton University Press, Princeton, 2005. 